$$
c_{q}\left(A_{q i i}-A_{q j i}\right)=0
$$

is a necessary condition for the permanence of vector-lines. It is also sufficient, as we see from consideration of the order of the equations involved and the fact that if (13) holds, then $\Omega_{i j}=0$ implies $\partial \Omega_{i j} / \partial t$ $=0$.

Hence we have this result: $A$ necessary and sufficient condition for the permanence of the vector-lines of $c_{i}$ is (13), or equivalently, that the tensor $c_{q} A_{q i j}$, that is,

$$
c_{j}\left(c_{i, k} v_{k}+\partial c_{i} / \partial t\right)+c_{i} v_{j, k} c_{k},
$$

shall be symmetric.

Dublin Institute for Advanced Studies

\title{
NOTE ON A THEOREM IN SUMMABILITY
}

J. D. HILL

Let $T$ denote a regular matrix method of summability in the complex domain, that is to say, a transformation of the form

$$
t_{n}=\sum_{k=1}^{\infty} a_{n k} s_{k} \quad(n=1,2,3, \cdots),
$$

having the property that the convergence of $\left\{s_{k}\right\}$ to $s$ always implies the existence of $t_{n}$ for each $n$ and the convergence of $\left\{t_{n}\right\}$ to $s$. It is well known that the following conditions of Silverman-Toeplitz are necessary and sufficient in order that $T$ be regular: $a_{n k}=o(1)(n \rightarrow \infty$; $k=1,2,3, \cdots) ; \sum_{k=1}^{\infty} a_{n k}=1+o(1)(n \rightarrow \infty) ;$ and

$$
\sum_{k=1}^{\infty}\left|a_{n k}\right|=O(1) \quad(n \rightarrow \infty) .
$$

The following theorem was established recently by Henstock [2].1

TheOREM (HeNstock). Let $y \equiv\left\{z_{k}\right\}$ be a given bounded sequence of complex numbers. Then there exist denumerably many sequences of

Received by the editors July $5,1950$.

${ }^{1}$ Numbers in brackets refer to the references at the end of the paper. 
zeros and ones, depending only on $y$, such that the summability of each of these by any regular matrix method $T$ implies the $T$-summability of $y$.

The proof given by Henstock follows classical lines and is moderately complicated. The purpose of this note is to give a shorter and simpler proof based on the theory of linear operations [1]. To this end we consider the sequences $X(t) \equiv\left\{\alpha_{k}\right\}$ of zeros and ones, with infinitely many ones, where $t \equiv \alpha_{1} \alpha_{2} \alpha_{3} \cdots(\operatorname{radix} 2)$. It is known [3] that the set of all sequences $X(t)$ constitutes a fundamental set in the Banach space (b) of all bounded complex sequences $x \equiv\left\{s_{k}\right\}$ with $\|x\| \equiv \sup _{k}\left|s_{k}\right|$. In other words, for $p=1,2,3, \cdots$ and an arbitrarily given $y \in(b)$ there exist a finite number of complex constants $A_{1}^{p}, A_{2}^{p}, \cdots, A_{m_{p}}^{p}$ and corresponding sequences $X\left(t_{1}^{p}\right), X\left(t_{2}^{p}\right), \cdots$, $X\left(t_{m_{p}}^{p}\right) \quad$ such that $\left\|y-\left[A_{1}^{p} X\left(t_{1}^{p}\right)+A_{2}^{p} X\left(t_{2}^{p}\right)+\cdots+A_{m_{p}}^{p} X\left(t_{m_{p}}^{p}\right)\right]\right\|$ $<1 / p$. If $y_{p}$ denotes the sequence in brackets we therefore have $y_{p} \rightarrow y$ in the norm of $(b)$. Let $T$ be a regular matrix method and write $f_{n}(x)=\sum_{k=1}^{\infty} a_{n k} s_{k}(n=1,2,3, \cdots)$ where $x=\left\{s_{k}\right\} \in(b)$. For each $n, f_{n}(x)$ is a linear operation on $(b)$ to the space of complex numbers, and $\left\|f_{n}\right\|=\sum_{k=1}^{\infty}\left|a_{n k}\right|$ [3]. If each of the denumerably many sequences $X\left(t_{i}^{p}\right)\left(i=1,2, \cdots, m_{p} ; p=1,2,3, \cdots\right)$ is summable- $T$, then the sequence $y_{p}$ is summable- $T$, that is, the sequence $\left\{f_{n}\left(y_{p}\right)\right\}$ is convergent for each $p$. Since the sequence of norms $\left\{\left\|f_{n}\right\|\right\}$ is bounded by (1) and $y_{p} \rightarrow y$, it follows (as in $[1$, p. 79, proof of Theorem $3]$ ) that the sequence $\left\{f_{n}(y)\right\}$ is convergent. This completes the proof.

\section{REFERENCES}

1. S. Banach, Théorie des opérations linéaires, Warsaw, 1932.

2. R. Henstock, The efficiency of matrices for bounded sequences, J. London Math. Soc. vol. 25 (1950) pp. 27-33.

3. H. J. Hamilton and J. D. Hill, Operation theory and multiple sequence transformations, Duke Math. J. vol. 8 (1941) pp. 154-162.

Michigan State College 